\title{
O impacto da pandemia SARS-CoV-2 no marketing da enfermagem: uma revisão
}

\section{integrativa}

\author{
The impact of the SARS-CoV-2 pandemic on nursing marketing: an integrative review \\ El impacto de la pandemia SARS-CoV-2 en el marketing de la enfermería: una revisión \\ integradora
}

\author{
Marcos José Vilchez David \\ ORCID: https://orcid.org/0000-0002-8794-1473 \\ Universidade Veiga de Almeida, Brasil \\ E-mail: marcosjvdavid@gmail.com \\ Juliana Lana Querino de Oliveira \\ ORCID: https://orcid.org/0000-0002-0472-7480 \\ Universidade Veiga de Almeida, Brasil \\ E-mail: juquerino3@gmail.com \\ Maria Virginia Godoy da Silva \\ ORCID: https://orcid.org/0000-0003-3980-042X \\ Universidade Veiga de Almeida, Brasil \\ E-mail: godoysilva@terra.com.br \\ Leandro Andrade da Silva \\ ORCID: https://orcid.org/0000-0003-3213-5527 \\ Universidade Veiga de Almeida, Brasil \\ E-mail: proflandrade@gmail.com \\ Roni Robson da Silva \\ ORCID: https://orcid.org/0000-0001-6010-6438 \\ Universidade Veiga de Almeida, Brasil \\ E-mail: rr.roni1@gmail.com \\ Milena Preissler das Neves \\ ORCID: https://orcid.org/0000-0002-3890-924X \\ Universidade Veiga de Almeida, Brasil \\ E-mail: milenapreissler@gmail.com
}

\begin{abstract}
Resumo
A pandemia pelo SARS-Cov-2 assombra o planeta desde o ano de 2020, dizimando milhares de pessoas em todo o mundo. Esse momento poderia ser propício para que o mundo reconhecesse o valor dos profissionais de saúde, do enfermeiro e da Enfermagem. Objetivo: Analisar as produções científicas sobre o impacto da pandemia pela SARSCov-2 no marketing da Enfermagem. Metodologia: Revisão integrativa da literatura, de acordo com a estratégia PICo e procedimentos definidos pelo Preferred Reporting Items for Systematic Reviews and Meta-Analyses (PRISMA). Bases de dados consultadas: Assia_Coronavírus; BVS; CINAHIL; Embase; PubMed; SciELO; Scopus e WOS. Resultados: Onze estudos foram selecionados para análise. A Enfermagem ganhou holofotes pela atuação. Os profissionais de enfermagem tiveram pouco acesso a Equipamentos de Proteção Individual (EPIs) adequados; faltaram espaços e recursos apropriados para a assistência e um subdimensionamento das equipes. O Brasil liderou o ranking mundial de óbitos de profissionais de enfermagem. O não reconhecimento do poder da profissão; altas jornadas de trabalho, salários baixos, resultam na desvalorização do trabalho do próprio enfermeiro Conclusão: Durante a pandemia pela SARS-CoV-2, a Enfermagem esteve na linha de frente, o que possibilitou de certa forma maior visibilidade profissional. As condições de trabalho desfavoráveis, baixos salários e outros desafios enfrentados pelos profissionais de enfermagem sinalizam que a profissão ainda carece de reconhecimento e que esforços para o marketing favorável precisam ser empenhados.
\end{abstract}

Palavras-chave: Enfermagem; Marketing; SARS-CoV-2; Pandemia.

\begin{abstract}
The SARS-Cov-2 pandemic has haunted the planet since the year 2020, decimating thousands of people around the world. This moment could be propitious for the world to recognize the value of health professionals, nurses and Nursing. Objective: To analyze scientific productions on the impact of the SARS-Cov-2 pandemic on Nursing marketing. Methodology: Integrative literature review, according to the PICo strategy and procedures defined by the Preferred Reporting Items for Systematic Reviews and Meta-Analyses (PRISMA). Databases consulted: Assia_Coronavirus; VHL; CINAHIL; base; PubMed; SciELO; Scopus and WOS. Results: Eleven studies were selected for analysis. Nursing gained the spotlight for its performance. Nursing professionals had little access to adequate Personal Protective Equipment (PPE); there was a lack of appropriate spaces and resources for assistance and an undersizing of the teams.
\end{abstract}


Brazil led the world ranking of deaths of nursing professionals. The non-recognition of the power of the profession; high working hours, low wages, result in the devaluation of the work of the nurse itself Conclusion: During the SARSCoV-2 pandemic, Nursing was on the front line, which allowed, in a way, greater professional visibility. The unfavorable working conditions, low wages and other challenges faced by nursing professionals indicate that the profession still lacks recognition and that efforts for favorable marketing need to be made.

Keywords: Nursing; Marketing; SARS-CoV-2; Pandemic.

\section{Resumen}

La pandemia del SARS-Cov-2 ha perseguido al planeta desde el año 2020, diezmando a miles de personas en todo el mundo. Este momento puede ser propicio para que el mundo reconozca el valor de los profesionales de la salud, las enfermeras y la Enfermería. Objetivo: Analizar las producciones científicas sobre el impacto de la pandemia del SARSCov-2 en el marketing de Enfermería. Metodología: Revisión integrativa de la literatura, según la estrategia PICo y los procedimientos definidos por los Preferred Reporting Items for Systematic Reviews and Meta-Analyses (PRISMA). Bases de datos consultadas: Assia_Coronavirus; BVS; CINAHILO; base; PubMed; SciELO; Scopus y WOS. Resultados: Once estudios fueron seleccionados para el análisis. La enfermería ganó protagonismo por su desempeño. Los profesionales de enfermería tenían poco acceso a Equipos de Protección Personal (EPP) adecuados; faltaron espacios y recursos apropiados para la atención y subdimensionamiento de los equipos. Brasil lideró el ranking mundial de muertes de profesionales de enfermería. El no reconocimiento del poder de la profesión; alta jornada laboral, bajos salarios, resultan en la desvalorización del trabajo del propio enfermero Conclusión: Durante la pandemia del SARSCoV-2, la Enfermería estuvo en primera línea, lo que permitió, en cierto modo, una mayor visibilidad profesional. Las condiciones de trabajo desfavorables, los bajos salarios y otros desafíos que enfrentan los profesionales de enfermería indican que la profesión aún carece de reconocimiento y que se deben realizar esfuerzos para una comercialización favorable.

Palabras clave: Enfermería; Márketing; SARS-CoV-2; Pandemia.

\section{Introdução}

A pandemia pelo SARS-Cov-2 assombra o planeta desde o ano de 2020. A doença ocasionada pelo novo coronavírus vem dizimando milhares de pessoas em todo o mundo. Com isso, a rotina dos serviços de saúde se transformou por completo.

As unidades de tratamento intensivo superlotaram, pacientes graves, quantidades insuficientes de equipamentos seriam componentes desse cenário de saúde desafiador. Para os profissionais de saúde as jornadas exaustivas, por vezes ultrapassaram limites humanos, como o cansaço, necessidades fisiológicas e emocionais (Oliveira, 2020).

A pandemia roubou a cena no ano de comemoração da Enfermagem, designado pela Organização das Nações Unidas por ocasião do bicentenário de nascimento de Florence Nightingale, precursora da Enfermagem Moderna. Esse momento seria propício para que o mundo reconhecesse o valor dos profissionais de saúde, do enfermeiro e da Enfermagem (Oliveira, 2020).

Este estudo trata do impacto da pandemia pelo SARS-Cov-2 para a Enfermagem, mais especificamente para o marketing da profissão. O marketing profissional da enfermagem sempre esteve em constante modificação (Cavalcanti, 2014). No início da profissão, o papel da enfermagem era exercido por mulheres leigas, mercenárias, subornáveis, prostitutas, religiosas e senhoras de caridade, bondosas, e que principalmente, não possuíam conhecimento científico (Costa et al.,2004).

A Enfermagem começou a se desenvolver a partir do século XIX, com Florence Nightingale, onde passou a aplicar o conhecimento científico relacionado ao cuidado com o cliente. Florence teve, como objetivo principal, afastar a imagem das enfermeiras leigas, preocupando-se principalmente com a origem socioeconômica e conduta moral das alunas e em estabelecer a imagem da enfermeira como anjo branco e submissa, onde mudou a figura que possuía antes a profissão (Gentil, 2009).

A história de uma carreira iniciada por mulheres sem qualificação técnica poderia explicar parte do conhecimento e reconhecimento da profissão de Enfermagem em nosso país e no mundo como um todo. No entanto, o marketing relacionado às profissões da saúde sempre foi um tema complexo. Ele é definido como uma função organizacional e um conjunto de processos que cria, comunica e agrega valor e gerência relação com o cliente, beneficiando dessa forma a organização e os seus stakeholders (Mendes et al., 2011).

Partindo do pressuposto de que a inabilidade da profissão valorizar seus profissionais ameaça a sua própria visibilidade, a Enfermagem precisa ter um posicionamento mais proativo neste cenário, aliando-se ao apoio de organismos internacionais e 
valendo-se de evidências para reivindicar melhores condições de trabalho e mais valorização. Deve mostrar o seu valor e sua competência para o sistema, para seus clientes, para as instituições e para a sociedade, ou seja, usar o marketing profissional, alcançando maior visibilidade social, através do resultado de seu trabalho (Mendes et al., 2011).

A partir do século XX e atualmente, a figura do enfermeiro e seu papel em relação ao usuário dos serviços de saúde modificaram em alguns aspectos. Parte dos profissionais demonstra maior preocupação com o desenvolvimento de uma postura mais humanizada e de acolhimento dos pacientes. No entanto, muitas vezes, está sobrecarregado pela carga de trabalho e horas excessivas de plantões (Cavalcanti, 2014). Essa seria uma caracterização semelhante ao atual momento vivido pelos profissionais de enfermagem do Brasil e de todo o mundo. Diante de tantos desafios enfrentados, seria a profissão valorizada e reconhecida? Esse estudo buscou atender a seguinte questão norteadora: Como a pandemia pela SARS-Cov-2 contribuiu para o marketing de enfermagem? Pretendeu-se contribuir com informações relevantes para o desenvolvimento do marketing pessoal na formação do enfermeiro. O objetivo deste estudo foi analisar as produções cientificas sobre o impacto da pandemia pelo SARS-Cov-2 no marketing da Enfermagem, bem como o estado da arte sobre a temática.

\section{Metodologia}

Trata-se de estudo de revisão integrativa da literatura, que possibilita a síntese e a conclusão geral do conhecimento científico de um determinado tema específico. Os componentes da revisão integrativa percorrem seis etapas: identificação do tema e seleção da hipótese ou questão de pesquisa; estabelecimento de critérios para inclusão e exclusão de estudos/ amostragem ou busca na literatura; definição das informações a serem extraídas dos estudos selecionados/ categorização dos estudos; avaliação dos estudos incluídos na revisão integrativa; interpretação dos resultados e apresentação da revisão/síntese do conhecimento (Mendes et al., 2008).

O estudo buscou atender a seguinte questão norteadora: Como a pandemia pela SARS-Cov-2 contribuiu para o marketing de enfermagem? Os principais assuntos foram identificados e sistematizados pelos elementos Participante, Interesse e Contexto, conhecido como estratégia PICo (Quadro 1), que é um acrônico adaptado pelo Joanna Briggs Institute (Joanna Briggs Institute, 2014).

Quadro 1: Aplicação da estratégia PICo, Rio de Janeiro, RJ, Brasil 2021.

\begin{tabular}{|c|c|c|}
\hline ACRÔNIMO & DEFINIÇÃO & APLICAÇÃO \\
\hline $\mathrm{P}$ & PARTICIPANTE & ENFERMAGEM \\
\hline $\mathrm{I}$ & INTERESSE & MARKETING \\
\hline $\mathrm{CO}$ & CONTEXTO & PANDEMIA \\
\hline
\end{tabular}

Fonte: Autores (2021).

Em seguida a seguinte estratégia de busca foi adotada com as seguintes palavras-chave ((enfermagem OR enfermeira OR enfermeir* OR enfermeros OR enfermeras) AND (marketing OR mercadotecnía OR publicidade OR publicidad OR promover OR promoção OR divulgação OR destaque OR protagonismo OR projeção OR projetar OR notícias OR televisão OR televisor*) AND ("novo coronavirus" OR "Covid-19" OR "2019-NCoV" OR "Coronavírus 2019-nCoV" OR covid-19 OR "covid 2019" OR "2019 novo coronavirus" OR "novo coronavírus (nCoV)" OR "novo coronavírus" OR covid2019 OR "infecção por coronavirus 2019" OR "nova infecção por coronavirus" OR "coronavírus 2019" OR "Nuevo coronavirus" OR "Coronavirus de Wuhan" OR pandemias OR pandemia)) AND ( db:("BDENF" OR "LILACS" OR "MULTIMEDIA" OR "PAHOIRIS" OR "IBECS" OR "LIS" OR "PREPRINT-SCIELO" OR "colecionaSUS") AND la:("pt" OR "es" OR "en")). 
Estas foram definidas a partir do vocabulário dos Descritores em Ciências da Saúde (DeCS), por ser uma terminologia comum à pesquisa. nas bases de dados e/ou bibliotecas eletrônicas. Para a construção das estratégias de busca foram utilizados nos termos as aspas (") para restringir termos compostos e estabelecer sua ordem.

Os operadores booleanos e/ou de pesquisa AND — interseção dos termos, OR — união dos termos compostos e/ou agrupamento dos sinônimos. Foram consultadas as bases de dados Sistema Online de Busca e Análise de Literatura Médica (MEDLINE), consultada via PubMed, Biblioteca Virtual em Saúde (BVS), Assia_Coronavírus, Embase, SciELO, Scopus e Web of Science (WOS), Cinahil. Os arquivos selecionados foram arquivados, removidas as duplicatas e exportados para uma planilha de edição do Excel, com os seguintes dados: número do artigo, autor, título, ano, volume/número/página, base de dados, resumo e palavras-chave.

Para a seleção dos artigos foram utilizados como como critérios de inclusão: artigos publicados entre 2016 e 2021 , disponíveis nos idiomas português, inglês e espanhol, na forma do artigo científico, com texto completo.

O estudo atendeu as etapas de elaboração recomendadas pelo Preferred Reporting Items for Systematic Reviews and Meta-Analyses (PRISMA). (GALVÃO, 2015) O processo de identificação, seleção, elegibilidade e inclusão está detalhado no fluxograma abaixo (Figura 1).

Figura 1: Fluxograma do processo de busca e seleção, Rio de Janeiro, RJ, Brasil, 2021.
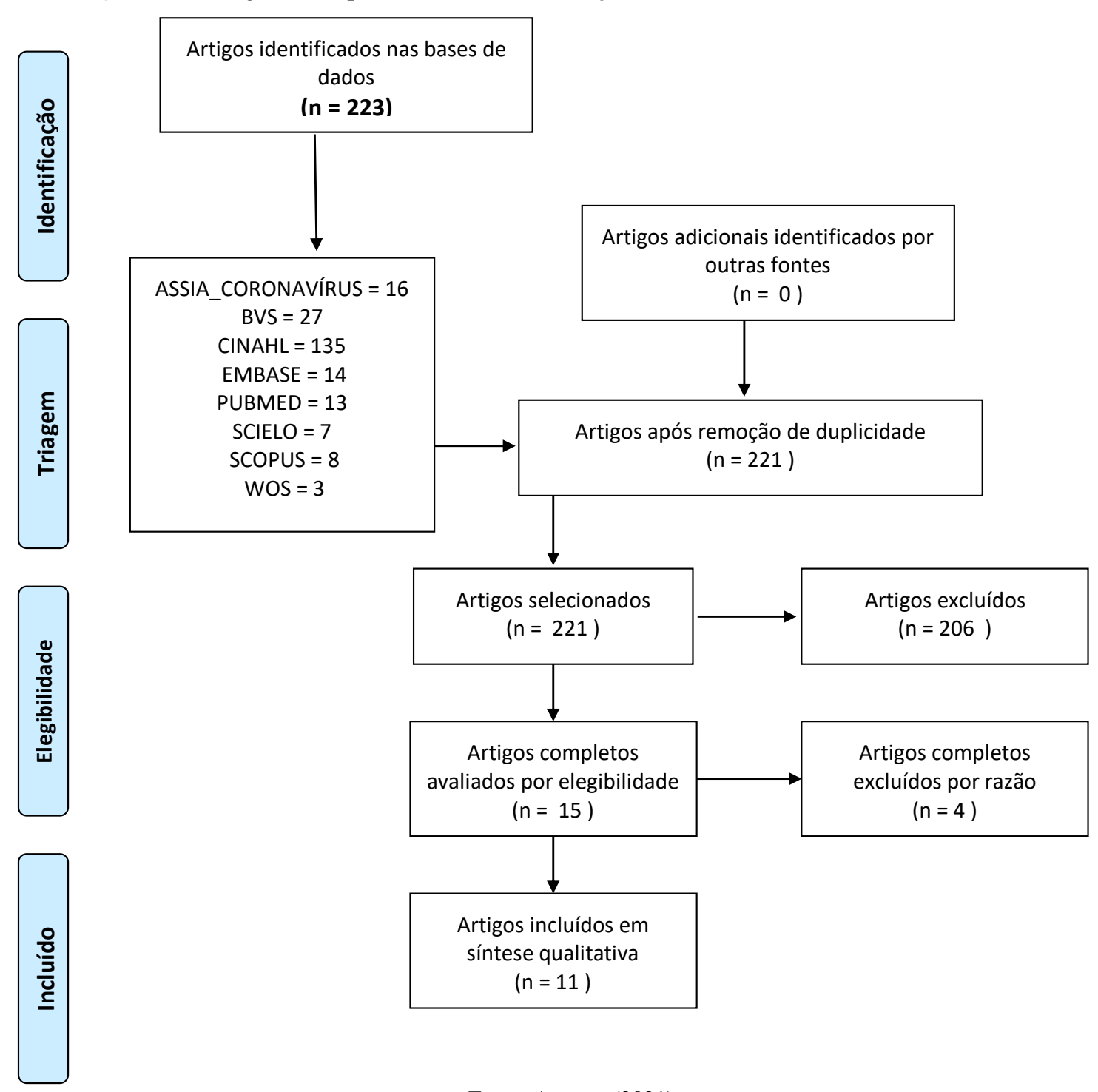

Fonte: Autores (2021). 


\section{Resultados e Discussão}

Dentre os onze estudos selecionados, dez foram publicados no Brasil, sendo um relato de experiência, um estudo analítico, um descritivo exploratório, um editorial e sete reflexivos. Não houve predominância de autores. Dez trabalhos foram publicados no ano de 2020 e um em 2021.

Quadro 2: Caracterização dos estudos que compuseram a amostra, segundo título, periódico, pais de origem, ano de publicação e resultados, Rio de Janeiro, RJ, Brasil, 2021.

\begin{tabular}{|c|c|c|c|}
\hline $\mathbf{N}^{\mathbf{o}}$ & Título / Autores & Periódicos / País / Ano & Resultados \\
\hline 1 & $\begin{array}{l}\text { "Pandemics, crisis conjunctures, and } \\
\text { professional practices: what is the role of } \\
\text { nursing with regard to Covid-19?" } \\
\text { David, HMSL; Acioli, SS; Silva, MRF; Bonetti, } \\
\text { OP; Passos, H. }\end{array}$ & $\begin{array}{c}\text { Rev. Gaúcha Enferm. / } \\
\text { Brasil / } 2021\end{array}$ & $\begin{array}{l}\text { Muitas dificuldades: pouco acesso aos EPIS adequados; } \\
\text { falta de espeço e recursos adequados para a } \\
\text { assistência; subdimensionamento das equipes; elevado } \\
\text { número de profissionais infectados e afastados pela } \\
\text { doença. O Brasil ocupa o primeiro lugar no ranking } \\
\text { mundial de óbitos de profissionais de enfermagem. } \\
\text { Sugestões: protagonizar discussões e mobilizações pelo } \\
\text { SUS; fortalecimento da atenção primária e combate à } \\
\text { precarização do trabalho em saúde. }\end{array}$ \\
\hline 2 & $\begin{array}{l}\text { "Nursing Now and the role of nursing in the } \\
\text { context of pandemic and current work" } \\
\text { Oliveira, KKD; Freitas, RJM; Araújo, JL; } \\
\text { Gomes, J GN. }\end{array}$ & $\begin{array}{l}\text { Rev. Gaúcha Enferm. } \\
\text { Brasil } \\
2020\end{array}$ & $\begin{array}{l}\text { Profissionais não reconhecem o poder da profissão; } \\
\text { altas jornadas de trabalho; salários baixos; } \\
\text { desvalorização do trabalho do enfermeiro. Sugestão: A } \\
\text { autovalorização é importante; ampliação das pesquisas } \\
\text { baseadas em evidências, o crescimento substancial de } \\
\text { estudos clínicos e do número de profissionais com } \\
\text { cursos de mestrado, doutorado e pós-doutorado. }\end{array}$ \\
\hline 3 & $\begin{array}{l}\text { "Protagonismo da Enfermagem Brasileira } \\
\text { no combate ao COVID-19" } \\
\text { Silva, MCN; Cunha, Carlos Leonardo } \\
\text { Figueiredo; Ximenes Neto, Francisco } \\
\text { Rosemiro Guimarães; Santos, José Luis } \\
\text { Guedes dos; Lourenção, Luciano Garcia; } \\
\text { Freire, Neyson Pinheiro; Cunha, Isabel } \\
\text { Cristina Kowal Olm. }\end{array}$ & $\begin{array}{l}\text { Enferm. foco } \\
\text { Brasil } \\
2020\end{array}$ & $\begin{array}{l}\text { Maior força de trabalho da área da saúde; ganhou } \\
\text { holofotes devido a atuação na pandemia da COVID-19; } \\
\text { espinha dorsal das equipes de saúde; a enfermagem } \\
\text { possui campo fértil de pesquisas e intervenções. } \\
\text { Sugestão: os profissionais de Enfermagem precisam ser } \\
\text { assegurados políticas e medidas governamentais que } \\
\text { contribuam para a prática segura e efetiva do cuidado. }\end{array}$ \\
\hline 4 & $\begin{array}{l}\text { "Comitê gestor de crise do coronavírus no } \\
\text { âmbito do Cofen" } \\
\text { Santos, VC; Persegona, MFM; Souza, EF; } \\
\text { Almeida, WC; Filete, MS; Silva, MCN. }\end{array}$ & $\begin{array}{l}\text { Enferm. foco } \\
\text { Brasil } \\
2020\end{array}$ & $\begin{array}{l}\text { Não apenas em razão de sua capacidade técnica, mas } \\
\text { também pela pluralidade. O COFEN desenvolveu ações } \\
\text { de enfrentamento a COVID com forma de proteção ao } \\
\text { profissional. }\end{array}$ \\
\hline 5 & $\begin{array}{l}\text { "Perspectivas e } \text { contribuições da } \\
\text { enfermagem para promover a saúde } \\
\text { universal Organização Pan-Americana da } \\
\text { Saúde" } \\
\text { OPAS }\end{array}$ & $\begin{array}{l}\text { PAHO-IRIS } \\
\text { EUA } \\
2020\end{array}$ & $\begin{array}{l}\text { A visibilidade no espaço nas produções acadêmicas da } \\
\text { área; partir desses resultados, a profissão já } \\
\text { demonstrou que possui sua própria voz em defesa das } \\
\text { políticas. }\end{array}$ \\
\hline 6 & $\begin{array}{l}\text { "Ano internacional da enfermagem e a } \\
\text { pandemia da covid-19: a expressão na } \\
\text { mídia" } \\
\text { Silva, João Miguel Almeida Ventura; Ribeiro, } \\
\text { Olga Maria Pimenta Lopes; Trindade, Letícia } \\
\text { Lima; Nogueira, Maria Assunção Almeida; } \\
\text { Monteiro, Maria Amélia José. }\end{array}$ & $\begin{array}{l}\text { Ciênc. cuid. saúde } \\
\text { Brasil } \\
2020\end{array}$ & $\begin{array}{l}\text { Falta de Equipamentos de Proteção Individual } \\
\text { (EPI); muitas pessoas infectadas no Brasil; medo do } \\
\text { profissional de infectar-se e transmitir o vírus; desgaste } \\
\text { físico e emocional; carência de enfermeiros nas } \\
\text { instituições de saúde; aprecariedade nos contratos de } \\
\text { trabalho; aumento da carga de trabalho gerando } \\
\text { aumento dos eventos adversos; reconhecimento pela } \\
\text { população dos profissionais. Sugestão: as associações } \\
\text { sindicais dando voz às preocupações dos enfermeiros. }\end{array}$ \\
\hline
\end{tabular}




\begin{tabular}{|c|c|c|c|}
\hline 7 & $\begin{array}{l}\text { "Pandemia do novo Coronavírus (SARS- } \\
\text { CoV-2): o protagonismo da enfermagem - } \\
\text { uma relação do passado com o presente e } \\
\text { perspectivas para o futuro" } \\
\text { Oliveira, Patricia Cristina Cavalari de. }\end{array}$ & $\begin{array}{c}\text { Nursing } \\
\text { Brasil } \\
2020\end{array}$ & $\begin{array}{l}\text { Enfermagem não tinha visibilidade social como a } \\
\text { observada atualmente. Evolução com total } \\
\text { embasamento científico para o cuidado de prática } \\
\text { baseada em evidências científicas. Profissionais de } \\
\text { enfermagem representam a maior categoria atuante } \\
\text { na área de saúde. Desafios: escassez de EPIS, } \\
\text { capacitação adequada das equipes, falta de } \\
\text { fluxos/protocolos, sobrecarga de trabalho, } \\
\text { subdimensionamento das equipes. Declaração da OMS: } \\
\text { a Enfermagem é sumamente importante no esforço } \\
\text { global para o alcance dos Objetivos De } \\
\text { Desenvolvimento Sustentável. Sugestões: aumentar o } \\
\text { financiamento para educar e empregar enfermeiros; } \\
\text { fortalecer a capacidade de coleta, análise e uso de } \\
\text { dados da força de trabalho em saúde }\end{array}$ \\
\hline 8 & $\begin{array}{l}\begin{array}{l}\text { "Reflexões acerca do exercício } \\
\text { supervisão da }\end{array} \\
\text { enfrentamento da covid-19" } \\
\text { enfermagem no } \\
\text { Chaves, Lucieli Dias Pedreschi; Fabro, Gisele } \\
\text { Caroline Richi; Galiano, Camila; Trovó, } \\
\text { Mayra de Cássia; Tomaz, Wanderson } \\
\text { Borges; Gleriano, Josué Souza. }\end{array}$ & $\begin{array}{c}\text { CuidArte, Enferm } \\
\text { Brasil } \\
2020\end{array}$ & $\begin{array}{l}\text { O enfermeiro exerce papel primordial, tendo a } \\
\text { supervisão de enfermagem como prática privativa, que } \\
\text { favorece atuar na interface das demandas dos } \\
\text { pacientes, do hospital e de equipe. Sugestão: buscar } \\
\text { estratégias para identificação de fatores que podem } \\
\text { caracterizar o adoecimento mental dos profissionais de } \\
\text { saúde diante dos desafios impostos. }\end{array}$ \\
\hline 9 & $\begin{array}{l}\text { "Nurse's protagonism in structuring and } \\
\text { managing a specific unit for covid-19" } \\
\text { Bitencourt, Julia Valeria de Oliveira Vargas; } \\
\text { Meschial, William Campo; Frizon, Gloriana; } \\
\text { Biffi, Priscila; Souza, Jeane Barros de; } \\
\text { Maestri, Eleine. }\end{array}$ & $\begin{array}{c}\text { Texto \& Contexto - } \\
\text { Enfermagem } \\
\text { Brasil } \\
2020\end{array}$ & $\begin{array}{l}\text { Crise atual setor de saúde diante da pandemia permitiu } \\
\text { mostrar a excelência do profissional enfermeiro; papel } \\
\text { fundamental desde a composição das comissões, } \\
\text { planejamento e funcionamento da estrutura física, } \\
\text { gestão de recursos humanos capacitados e construção } \\
\text { de protocolos e fluxos de cuidado, além de atuarem } \\
\text { diretamente na assistência. }\end{array}$ \\
\hline 10 & $\begin{array}{l}\text { "200 Years of Florence and the challenges of } \\
\text { nursing practices management in the } \\
\text { COVID-19 pandemic" } \\
\text { Geremia, Daniela Savi; Vendruscolo, Carine; } \\
\text { Celuppi, Ianka Cristina; Adamy, Edlamar } \\
\text { Kátia; Toso, Beatriz Rosana Gonçalves de } \\
\text { Oliveira; Souza, Jeane Barros de. }\end{array}$ & $\begin{array}{c}\text { Revista Latino- } \\
\text { Americana de } \\
\text { Enfermagem Brasil } \\
2020\end{array}$ & 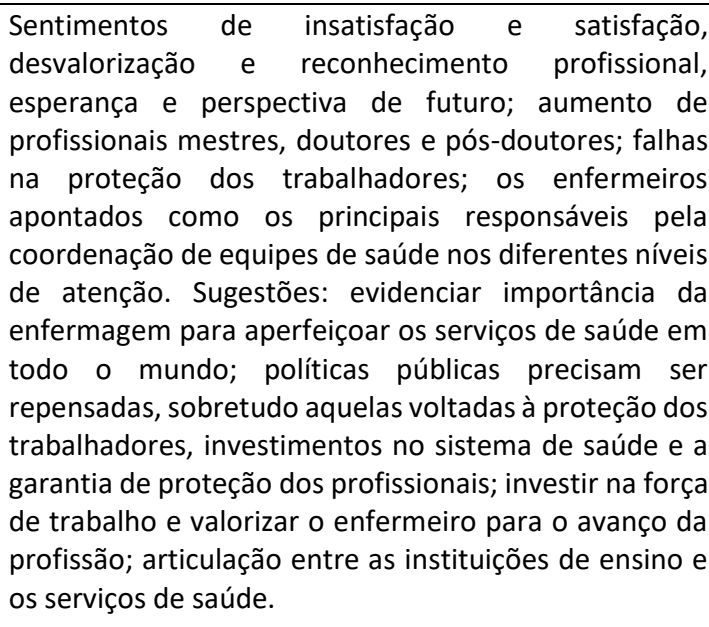 \\
\hline 11 & $\begin{array}{l}\text { "Challenges faced by pediatric nursing } \\
\text { workers in the face of the COVID-19 } \\
\text { pandemic" } \\
\text { Góes, Fernanda Garcia Bezerra; Silva, Aline } \\
\text { Cerqueira Santos Santana da; Santos, } \\
\text { Andressa Silva Torres dos; Pereira-Ávila, } \\
\text { Fernanda Maria Vieira; Silva, Laura } \\
\text { Johanson da; Silva, Liliane Faria da; Goulart, } \\
\text { Maithê de Carvalho e Lemos. }\end{array}$ & $\begin{array}{l}\text { Revista Latino- } \\
\text { Americana de } \\
\text { Enfermagem } \\
\text { Brasil } \\
2020\end{array}$ & $\begin{array}{l}\text { Ausência de treinamentos, de testes diagnósticos e de } \\
\text { conhecimentos/informações relacionados à COVID-19. } \\
\text { Número reduzido dos profissionais de Enfermagem. } \\
\text { Desvalorização da categoria. Crise relacionada à } \\
\text { provisão de materiais essenciais e de qualidade para a } \\
\text { prevenção e o enfrentamento da doença, tais como } \\
\text { testes diagnósticos e EPIs, impactando diretamente a } \\
\text { segurança do cuidado e do trabalho em saúde. }\end{array}$ \\
\hline
\end{tabular}

Fonte: Autores (2021).

As categorias de análise foram organizadas em três vertentes: Sem Protagonismo, Protagonismo Débil e Protagonismo Presente. A primeira, denominada Sem Protagonismo reúne as opiniões e achados de autores que não concordam com o 
protagonismo da Enfermagem nessa pandemia pelo COVID-19. A segunda, denominada Protagonismo Débil aborda uma condição intermediária com alguns destaques para essa possível condição. A terceira categoria foi denominada Protagonismo Presente e engloba opiniões que ratificam a condição de destaque para a Enfermagem durante a pandemia.

Estudos apontaram para a falta de protagonismo (David et al., 2021); (Silva, JMAV et al.,2020); (Goes., 2020). Essa falta de protagonismo foi justificada por uma série de condições. Os profissionais de enfermagem têm pouco acesso aos Equipamentos de Proteção Individual (EPIs) adequados; faltam espaços e recursos adequados para a assistência e há um subdimensionamento das equipes. David et al., (2021) destacam as consequências para esses problemas: o elevado número de profissionais infectados e afastados pela doença levando o Brasil a ocupar o primeiro lugar no ranking mundial de óbitos de profissionais de enfermagem. $\mathrm{O}$ medo do profissional de se infectar gera desgaste físico e emocional; carência de enfermeiros nas instituições de saúde, assim como a precariedade nos contratos de trabalho e aumento da carga de trabalho gerando aumento dos eventos adversos (Silva, JMAV et al., 2020). (Goes et al.,2020).

As implicações para os graves problemas que caracterizaram a falta de protagonismo foram apresentadas também e mais especificamente com relação aos desafios da Enfermagem pediátrica. O déficit de funcionários e treinamentos direcionados a COVID-19 aos profissionais da saúde, gera um receio da contaminação/contágio de si e dos familiares. Cerca de $20 \%$ das infecções em crianças apresentam-se como uma ocorrência assintomática, potencializando a transmissibilidade causada pelo novo Coronavírus frente ao contato mais próximo entre o portador do vírus com o profissional de saúde e seus familiares (Goes et al.,2020).

Um único estudo trouxe uma categoria intermediária com relação ao protagonismo, sendo assim denominada Protagonismo Débil (Oliveira, KKD et al., 2020). Essa condição foi justificada pelo fato de os próprios profissionais de enfermagem não reconhecerem o poder da profissão; submeterem-se à altas jornadas de trabalho, salários baixos, desvalorizando o trabalho do próprio enfermeiro (Goes et al.,2020).

O protagonismo foi defendido de forma entusiasta pela maioria dos estudos selecionados. Segundo dados do COFEN, há mais de dois milhões de profissionais de enfermagem presentes em todos os municípios do Brasil, atuando em todos os níveis de atenção à saúde. A enfermagem é a maior força de trabalho da área da saúde, a espinha dorsal das equipes de saúde. Com a pandemia da COVID-19, ganhou holofotes devido à grande atuação, gerando assim, um protagonismo (Silva, MCN et al., 2020).

Dentre os estudos que destacam o protagonismo, considerou-se o momento atual oportuno para a enfermagem brasileira alavancar sua visibilidade, demonstrando competência frente ao cenário enfrentado. Para alcançar condições laborais saudáveis, e exercer a profissão com plenitude e segurança, impera a necessidade de persistir na luta por melhores condições de trabalho e pelo reconhecimento da essencialidade da enfermagem nos serviços de saúde. A jornada ideal de trabalho da enfermagem ainda não é regulamentada em lei e varia entre 30 horas e 40 a 44 horas semanais, sendo que a elevada carga de trabalho pode interferir na qualidade de vida, inviabilizando outras atividades: físicas, culturais, sociais, as quais são essenciais para se promover a saúde dos profissionais. O cenário atual tem valorizado a enfermagem pelo protagonismo na construção do seu corpo de conhecimento, na proatividade de organização dos cuidados e do SUS, na capacidade de liderança e no desenvolvimento de saberes que embasam os conhecimentos em evidências científicas (Bitencourt et al., 2020).

Vale destacar que os enfermeiros aproveitaram a oportunidade oferecida pelo reconhecimento da OMS de 2020 como o Ano Internacional da Enfermagem e da Obstetrícia para impulsionar as iniciativas em potenciar sua voz em matéria de pautas e políticas no futuro. E à próxima geração de enfermeiros, garantir que não apenas nos concentremos nas questões clínicas, mas também em diversificar e intensificar a nossa contribuição para esses determinantes mais amplos da saúde (OPAS, 2020).

Para a proteção profissional, o COFEN desenvolveu ações de enfrentamento a COVID. As medidas lançadas incluíram a criação de Juntos contra o Coronavírus, com o objetivo de concentrar e propagar informações corretas e atualizadas sobre o tema com o intuito de ampliar a segurança para a atuação dos profissionais. No Observatório da Enfermagem, foram concentradas 
informações sobre a evolução da COVID-19 nos profissionais de enfermagem do Brasil. As Diretrizes para a Fiscalização nos estados e municípios, incluíram o levantamento situacional de riscos relacionados à COVID-19. O setor de Ouvidoria e Denúncias, teve o intuito de ouvir os profissionais da enfermagem. Foi realizada a compra emergencial de máscaras N95/ PFF2. Para o apoio em Saúde Mental, foi disponibilizado um canal de atendimento que funciona 24 horas por dia, todos os dias da semana. A capacitação profissional, de forma gratuita e virtualizada foi oferecida pelo Plantão do Comitê de Crise a fim de acompanhar em tempo real as mudanças da pandemia (Santos et al., 2020).

\section{Considerações Finais}

Durante a pandemia pela SARS-CoV-2, a Enfermagem esteve na linha de frente, o que possibilitou de certa forma maior visibilidade profissional. Os estudos selecionados nessa revisão apontaram, na sua maioria, o protagonismo da Enfermagem. Esse protagonismo foi justificado pela participação majoritária nas equipes de saúde, o tempo de permanência na assistência à clientela. Porém foram destacados graves problemas que são enfrentados pelos profissionais não somente durante a pandemia. As condições de trabalho desfavoráveis, baixos salários dos profissionais de enfermagem sinalizam que a profissão ainda carece de reconhecimento e que esforços para o marketing favorável precisam ser empenhados. Cabe destacar ainda a crise relacionada à provisão de materiais essenciais e de qualidade para a prevenção e o enfrentamento da doença, tais como testes diagnósticos e EPIs, impactando diretamente a segurança do cuidado e do trabalho em saúde. Dessa forma, a revisão pode identificar as diferentes nuances do protagonismo incluindo a sua inexistência.

\section{Referências}

Bitencourt, J. V. D. O. V., Meschial, W. C., Frizon, G., Biffi, P., Souza, J. B. D., \& Maestri, E. (2020). Nurse's protagonism in structuring and managing a specific unit for Covid-19. Texto \& Contexto-Enfermagem, 29. https://doi.org/10.1590/1980-265X-TCE-2020-0213

Cavalcanti, M. C. S., Mariano, R. F., Chaves, R. G. R., Cavalcanti, V. F. S., \& Barbosa, W. F. (2014). A evolução da enfermagem: um recorte histórico, político e cultural. Congr Bras Cons Enferm, 10-10. https://livrozilla.com/doc/763375/a-evolu\%C3\%A7\%C3\%A3o-da-enfermagem--um-recorte

Chaves, L. D. P., Fabro, G. C. R., Galiano, C., Trovó, M. D. C., Tomaz, W. B., \& Gleriano, J. S. (2020). Reflexões acerca do exercício da supervisão de enfermagem no enfrentamento da COVID-19. CuidArte, Enferm, 10-17. https://pesquisa.bvsalud.org/portal/resource/pt/biblio-1118508

Costa, M. S., Forte, B. P., Alves, M. D. S., Viana, J. F., \& Oriá, M. O. B. (2004). Auditoria em enfermagem como estratégia de um marketing profissional. Revista Brasileira de Enfermagem, 57, 497-499. DOI: https://doi.org/10.1590/S0034-71672004000400024

Silva, R. R., Lessa de Souza, M. V., Ferracini Alencar, I., Ferreira Leite Inácio, A., Ferreira da Silva, D., Ferro Messias, I., \& Lemos de Magalhães, A. F. (2021). Neuropatias diabéticas periféricas como complicações do diabetes mellitus: estudo de revisão. Saúde Coletiva (Barueri), 11(67), 6923-6936. https://doi.org/10.36489/saudecoletiva.2021v11i67p6923-6936

David, H. M. S. L., Acioli, S., Silva, M. R. F. D., Bonetti, O. P., \& Passos, H. (2020). Pandemics, crisis conjunctures, and professional practices: what is the role of nursing with regard to Covid-19?. Revista Gaúcha de Enfermagem, 42. (https://doi.org/10.1590/1518-8345.4550.3367

Galvão TF, Pansani TSA, Harrad D. Principais itens para relatar revisões sistemáticas e meta-análises: a recomendação PRISMA. Epidemiol Serv Saúde. 2015;24(2):335-41. https://doi.org/10.5123/ S1679-49742015000200017

Gentil, R. C. (2009). O enfermeiro não faz marketing pessoal: a história explica por quê?. Revista Brasileira de Enfermagem, 62, 916-918. https://doi.org/10.1590/S0034-71672009000600019

Geremia, D. S., Vendruscolo, C., Celuppi, I. C., Adamy, E. K., Toso, B. R. G. D. O., \& Souza, J. B. D. (2020). 200 Years of Florence and the challenges of nursing practices management in the COVID-19 pandemic. Revista latino-americana de enfermagem, 28. https://doi.org/10.1590/1518-8345.4576.3358

Góes, F. G. B., Silva, A. C. S. S. D., Santos, A. S. T. D., Pereira-Ávila, F. M. V., Silva, L. J. D., Silva, L. F. D., \& Goulart, M. D. C. (2020). Challenges faced by pediatric nursing workers in the face of the COVID-19 pandemic. Revista Latino-Americana de Enfermagem, 28. https://doi.org/10.1590/15188345.4550 .3367

Joanna Briggs Institute. (2014). Joanna Briggs Institute reviewers' manual: 2014 edition. Australia: The Joanna Briggs Institute. https://nursing.lsuhsc.edu/JBI/docs/ReviewersManuals/Economic.pdf

Martins, M. J. R., \& Fernandes, S. J. D. (2014). A visibilidade da enfermagem, dando voz à profissão: revisão integrativa. Revista de Enfermagem UFPE On Line, 8(s1). DOI: 10.5205/reuol.5927-50900-1-SM.0807supl20143.

Mendes KDS, Silveira RCCP, Galvão CM. Revisão Integrativa: Método de pesquisa para a incorporação de evidências na saúde e na Enfermagem. Texto Contexto Enferm, Florianópolis, 2008 Out-Dez; 17(4): 758-64. 
Mendes, I. A. C., Trevizan, M. A., Mazzo, A., Godoy, S. D., \& Ventura, C. A. A. (2011). Marketing profissional e visibilidade social na enfermagem: uma estratégia de valorização de recursos humanos. Texto \& Contexto-Enfermagem, 20, 788-795. https://doi.org/10.1590/S0104-07072011000400019

Oliveira, A. C. (2020). Desafios da enfermagem frente ao enfrentamento da pandemia da Covid19. REME rev. min. enferm, e-1302. https://doi.org/10.5935/1415-2762.20200032

Oliveira, K. K. D. D., Freitas, R. J. M. D., Araújo, J. L. D., \& Gomes, J. G. N. (2020). Nursing Now and the role of nursing in the context of pandemic and current work. Revista Gaúcha de Enfermagem, 42. https://doi.org/10.1590/1983-1447.2021.20200120

Oliveira, P. C. C. (2020). Pandemia do novo Coronavírus (SARS-CoV-2): o protagonismo da enfermagem-uma relação do passado com o presente e perspectivas para o futuro. Nursing (São Paulo), 23(265), 4257-4268. https://doi.org/10.36489/nursing.2020v23i265p4257-4268

Organização Pan-Americana de Saúde. Perspectivas e Contribuições da Enfermagem para promover s saúde universal. 2020

Santos, V. C., Persegona, M. F. M., de Souza, E. F., Almeida, W. C., Filete, M., \& da Silva, M. C. N. (2020). Comitê gestor de crise do coronavírus no âmbito do Cofen. Enfermagem em Foco, 11(2). https://doi.org/10.21675/2357-707X.2020.v11.n2.4213

Silva, J. M. A. V., Ribeiro, O. M. P. L., Trindade, L. L., Nogueira, M. A. A., \& Monteiro, M. A. J. (2020). Ano internacional da enfermagem e a pandemia da covid-19: a expressão na mídia. Ciênc. cuid. saúde, 8-8. 10.4025/cienccuidsaude.v19i0.55546

Silva, LA da ., Soares, JPA, Silva, LF da, Silva, RR da, Araújo, MS ., Silva, MVG da ., Oliveira, ES de ., Mesquita, LMF de ., Alencar, Ícaro F. de, Dutra, V. de C. de A. ., Silveira, MB ., Norbiato, V. do N. de P. ., Silveira, MLFG da ., Souza, M. das GG de ., Souza, DAC., Barbosa, I. de SF ., Mendonça, C. de F. de S. ., Duque, E. da S. ., Oliveira, JVE ., \& Silva, SFM . (2021). Pandemias e suas repercussões sociais ao longo da história associadas ao novo SARS-COV-2: Um estudo de revisão. Pesquisa, Sociedade e Desenvolvimento , 10 (3), e59110313783. https://doi.org/10.33448/rsd-v10i3.13783

Silva, M. C. N., Cunha, C. L. F., Neto, F. R. G. X., dos Santos, J. L. G., Lourenção, L. G., Freire, N. P., \& Cunha, I. C. K. O. (2020). Protagonismo da Enfermagem Brasileira no combate ao COVID-19. Enfermagem em Foco, 11(1. ESP). https://doi.org/10.21675/2357-707X.2020.v11.n1.ESP.4073

Silva, R. R. da, \& Silva, L. A. da. (2021). Psychosocial load and burnout syndrome in healthcare professionals in the fight against COVID-19 pandemic / Carga psicossocial e síndrome de burnout em profissionais de saúde no combate a pandemia de COVID-19. Revista de Pesquisa Cuidado é Fundamental Online, 13, 1640-1646. https://doi.org/10.9789/2175-5361.rpcfo.v13.11097

Silva, R. R. da, Lipari, C. da C., Araujo, M. S., Silva, L. A. da, Silva, M. V. G. da, Franco, A. S., Marta, C. B., Larrubia, E. de O., Francisco, M. T. R., \& Oliveira, E. S. de. (2021). Contribuições da Monitoria em Fundamentos de Enfermagem II na Formação Acadêmica de Estudantes de Enfermagem: Relato de Experiência. Global Academic Nursing Journal, 2(1). https://doi.org/10.5935/2675-5602.20200079

Silva, R. R. da, Neves, M. P. das, Silva, L. A. da, Silva, M. V. G. da, Hipolito, R. L., \& Marta, C. B. (2020). Consumo de Drogas Psicoativas em Contexto de Sexual entre Homens Gays como Fator de Risco para Transmissão de HIV/Aids. Global Academic Nursing Journal, 1(3). https://doi.org/10.5935/26755602.20200057

Silva, R. R. da, Silva, L. A. da, Silva, M. V. G. da, Neves, M. P. das, Silva, M. M. dos S. da, Francisco, M. T. R., \& Marta, C. B. (2019). Os impactos do Chemsex na saúde pública mundial: um estudo sobre uma perigosa prática sexual entre homens. Saúde Coletiva (Barueri), 51, 1920-1925. https://doi.org/10.36489/saudecoletiva.2019v9i51p1920-1925

Silva, R. R. da, Silva, L. A. da, Souza, M. V. L. de, Silva, M. V. G. da, Neves, M. P. das, Vargas, D. de, Hipolito, R. L., Souza, D. A. C., Dutra, V. de C. de A., Oliveira, E. S. de, Lipari, C. da C., Garcia, W., Cortes, T., \& Mattos, C. M. (2021). Estresse de minoria de gênero e seus efeitos na saúde mental como fator de risco para depressão em pessoas transgênero: Revisão da literatura. Research, Society and Development, 10(3), e51610313693. https://doi.org/10.33448/rsdv10i3.13693

Silva, R. R., Pontes, L. G. de, Oliveira, G. A. de, Assmann, T. C., Campos, E. da C., Silva, A. A. da, \& Souza, M. V. L. de. (2021). Avaliação dos fatores de risco e diagnóstico para neuropatia autonômica cardíaca em pessoas diabéticas. Global Academic Nursing Journal, 2(nspe3). https://doi.org/10.5935/26755602.20200164 